\title{
NONEXISTENCE OF NODAL SOLUTIONS OF ELLIPTIC EQUATIONS WITH CRITICAL GROWTH IN $\mathbb{R}^{2}$
}

\author{
ADIMURTHI AND S. L. YADAVA
}

Abstract. Let $f(t)=h(t) e^{b t^{2}}$ be a function of critical growth. Under a suitable assumption on $h$, we prove that

$$
\begin{array}{cl}
-\Delta u=f(u) & \text { in } B(R) \subset \mathbb{R}^{2}, \\
u=0 & \text { on } \partial B(R),
\end{array}
$$

does not admit a radial solution which changes sign for sufficiently small $R$.

\section{INTRODUCTION}

Let $B(R)$ denote the ball of radius $R$ in $\mathbb{R}^{2}$ with center at zero. Let $f(t)=$ $h(t) e^{b t^{2}}$ be a function of critical growth (see Adimurthi-Yadava [1]). Consider the following problem

$$
\left\{\begin{aligned}
-\Delta u & =f(u) & & \text { in } B(R), \\
u & =0 & & \text { on } \partial B(R) .
\end{aligned}\right.
$$

If $f$ satisfies the following condition

$$
\lim _{t \rightarrow \infty} \frac{\log h(t)}{t}=\infty
$$

then (1.1) admits an infinite number of radial solutions which change sign (see Adimurthi-Yadava [1]).

In this note we show that the condition (1.2) is optimal for existence of infinitely many radial solutions which change sign by proving the following:

Theorem 1. Let $f(t)=t|t|^{m} e^{b t^{2}+|t|^{\beta}}, m \geq 0, b>0$ and $0 \leq \beta \leq 1$. Then for every $\beta$ there exists $R^{(\beta)}>0$ such that for $0<R<R^{(\beta)}$, the problem

$$
\left\{\begin{aligned}
-\Delta u & =f(u) & & \text { in } B(R), \\
u & =0 & & \text { on } \partial B(R),
\end{aligned}\right.
$$

does not admit any radial solution which changes sign.

If $1<\beta<2$, then $f$ satisfies (1.2) and hence (1.2) is optimal.

In this connection similar results are available for critical exponent problems in $\mathbb{R}^{n}, n \geq 3$. There the dimension plays a role in the case of existence

Received by the editors February 22, 1989 and, in revised form, April 2, 1990.

1980 Mathematics Subject Classification (1985 Revision). Primary 35J25; Secondary 35P30. 
(see Cerami-Solomini-Struwe [5]) and nonexistence (see Atkinson-Brezis-Peletier [4]) of radial solutions which change sign.

\section{Proof of Theorem 1}

Since we are looking for radial solutions, (1.3) becomes

$$
\left\{\begin{array}{l}
-\left(u^{\prime \prime}+\frac{1}{r} u^{\prime}\right)=f(u) \quad \text { in }(0, R), \\
u^{\prime}(0)=u(R)=0
\end{array}\right.
$$

By studying the following initial value problem we will prove the nonexistence of nodal solutions of (2.1) as in Atkinson-Brezis-Peletier [4]

$$
\left\{\begin{array}{l}
-\left(u^{\prime \prime}+\frac{1}{r} u^{\prime}\right)=f(u), \\
u^{\prime}(0)=0 \\
u(0)=\gamma>0 .
\end{array}\right.
$$

Let $R_{k}(\gamma), k=1,2, \ldots$, denote the $k$ th zero of $u$. Then by the similar argument as in Atkinson-Peletier [3] we have

$$
\lim _{\gamma \rightarrow 0} R_{1}(\gamma)= \begin{cases}\infty & \text { if } m>0, \\ C & \text { if } m=0,\end{cases}
$$

where $C$ is some positive constant. For the sake of completeness we will sketch the proof of (2.3) in Appendix 2. Now the proof of the theorem follows from the following:

Claim 1. For each $0 \leq \beta \leq 1$, there exists a constant $c(\beta)>0$ such that

$$
\lim _{\gamma \rightarrow \infty} R_{2}(\gamma)>c(\beta)
$$

In order to prove Claim 1, make the standard substitution (as in AtkinsonPeletier [2]) by $r=2 e^{-t / 2}$ and $u(r)=y(t)$, then (2.2) becomes

$$
\left\{\begin{array}{l}
-y^{\prime \prime}=e^{-t} f(y) \\
y(\infty)=\gamma \\
y^{\prime}(\infty)=0
\end{array}\right.
$$

Let $y(t, \gamma)$ be the corresponding solution and $T_{k}(\gamma)$ the $k$ th zero of $y(t, \gamma)$. Then

$$
R_{k}(\gamma)=2 e^{-T_{k}(\gamma) / 2}
$$

Now we have the following estimates on $T_{1}(\gamma)$.

Claim 2. For every $\beta, 0 \leq \beta \leq 1$, there exist constants $C_{\beta}>0$ and $\gamma_{0}>0$ such that for all $\gamma \geq \gamma_{0}$,

$$
\begin{gathered}
\gamma y^{\prime}\left(T_{1}(\gamma), \gamma\right) \leq C_{\beta}, \\
\frac{T_{1}(\gamma)}{\gamma} \leq C_{\beta}, \\
\lim _{\gamma \rightarrow \infty} T_{1}(\gamma)=\infty .
\end{gathered}
$$


Proof of Claim 1. Assuming Claim 2 we will complete the proof of Claim 1. Without loss of generality we may assume

$$
\lim _{\gamma \rightarrow \infty} T_{2}(\gamma) \geq 1
$$

By using the convexity of $y$ on $\left[T_{2}(\gamma), T_{1}(\gamma)\right]$ together with $(2.7)$ and (2.8) we have for all $\gamma \geq \gamma_{0}$ and $t \in\left[T_{2}(\gamma), T_{1}(\gamma)\right]$,

$$
|y(t, \gamma)| \leq\left|T_{1}(\gamma) y^{\prime}\left(T_{1}(\gamma), \gamma\right)\right| \leq\left|\frac{T_{1}(\gamma)}{\gamma} \gamma y^{\prime}\left(T_{1}(\gamma), \gamma\right)\right| \leq C_{\beta}^{2} .
$$

Let

$$
K(\beta)=\sup \left\{\frac{f(y)}{y}: 0 \leq y \leq C_{\beta}^{2}\right\}
$$

and choose $t_{0}(\beta)>0$ such that for $t \geq t_{0}(\beta)$,

$$
4 t^{2} e^{-t} K(\beta)<1 \text {. }
$$

From $(2.8)^{\prime}$, we can choose a $\gamma_{1}>\gamma_{0}$ such that for all $\gamma \geq \gamma_{1}$,

$$
t_{0}(\beta)<T_{1}(\gamma) \text {. }
$$

Hence from (2.10), (2.11) and (2.12) for all $t \geq t_{0}(\beta), t \in\left[T_{2}(\gamma), T_{1}(\gamma)\right]$, $\gamma \geq \gamma_{1}$, we have

$$
4 t^{2} e^{-t} \frac{f(y(t, \gamma))}{y(t, \gamma)}<1
$$

Let $Z=t^{1 / 2}$, then $Z$ satisfies

$$
Z^{\prime \prime}+\frac{1}{4 t^{2}} Z=0
$$

and

$$
y^{\prime \prime}+\frac{1}{4 t^{2}}\left(4 t^{2} e^{-t} \frac{f(y)}{y}\right) y=0 .
$$

Hence from (2.14) and by Sturm's Comparison Theorem we have for all $\gamma \geq \gamma_{1}$,

$$
T_{2}(\gamma)<t_{0}(\beta) \text {. }
$$

Now (2.4) follows from (2.6) and (2.17). This completes the proof of Claim 1 and hence Theorem 1.

In order to prove Claim 2 we need the following proposition.

Let $F: \overline{\mathbb{R}}_{+} \rightarrow \overline{\mathbb{R}}_{+}$be a locally Lipschitz continuous function and $s_{0} \geq 0$ such that

$$
F(s) \text { is strictly increasing for } s \geq s_{0} \text {. }
$$

$$
\text { Let } G(s)=\log F(s) \text { be } C^{2} \text { and convex for } s \geq s_{0} \text {. }
$$

$$
\left(\gamma G^{\prime}(\gamma)\right)^{2} e^{-\left\{G(\gamma)-\frac{1}{2}\left(\gamma-s_{0}\right) G^{\prime}(\gamma)\right\}}=O(1) \quad \text { as } \gamma \rightarrow \infty
$$

$$
\lim _{\gamma \rightarrow \infty} \frac{\gamma G^{(p+1)}(\gamma)}{G^{(p)}(\gamma)}=L_{p} \neq 0 \quad \text { for } p=0,1,
$$


where $G^{(p)}$ denotes the $p$ th derivative of $G$.

There exist positive constants $C_{1}, C_{2}, l$ and $\gamma_{1}$ such that for all $\gamma \geq \gamma_{1}$,

$$
C_{1} \gamma^{l} \leq G(\gamma) \leq C_{2} \gamma^{l}
$$

Let $Y(t, \gamma)$ denote the solution of

$$
\left\{\begin{array}{l}
-Y^{\prime \prime}=e^{-t} F(Y), \\
Y(\infty)=\gamma \\
Y^{\prime}(\infty)=0
\end{array}\right.
$$

and $S(\gamma)$ the first zero of $Y(t, \gamma)$. Let $S_{0}(\gamma)$ be such that $Y\left(S_{0}(\gamma), \gamma\right)=s_{0}$. Note that $S(\gamma) \leq S_{0}(\gamma)$. Then we have the following:

Proposition 2. We have, as $\gamma \rightarrow \infty$,

$$
\begin{aligned}
& Y^{\prime}\left(S_{0}(\gamma), \gamma\right) \\
& =\frac{2}{G^{\prime}(\gamma)}\left[1+O\left(\frac{(\log \gamma)^{2}}{G(\gamma)}\right)+O\left(\gamma G^{\prime}(\gamma) e^{-\left\{G(\gamma)-\frac{1}{2}\left(\gamma-s_{0}\right) G^{\prime}(\gamma)\right\}}\right)\right], \\
& S_{0}(\gamma)=\left(G(\gamma)-\frac{1}{2} \gamma G^{\prime}(\gamma)\right)+s_{0}\left(\frac{G^{\prime}(\gamma)}{2}\right)+\log \frac{G^{\prime}(\gamma)}{2} \\
& \quad+O((\log \gamma))^{2}+O\left[\left(\gamma G^{\prime}(\gamma)\right)^{2} e^{-\left\{G(\gamma)-\frac{1}{2}\left(\gamma-s_{0}\right) G^{\prime}(\gamma)\right\}}\right], \\
& S(\gamma) \geq\left(G(\gamma)-\frac{1}{2} \gamma G^{\prime}(\gamma)\right)+\log \frac{G^{\prime}(\gamma)}{2}+O(1) .
\end{aligned}
$$

Proof of this proposition follows exactly as in Atkinson-Peletier [2] (see Lemma 10 and Theorem 4). Since the hypotheses here on $G$ are little bit different from those in Atkinson-Peletier [2] we shall for completeness sketch the proof in Appendix 1.

Proof of Claim 2. Let $F(s)=s|s|^{m} e^{b s^{2}+|s|^{\beta}}$, then for $s \geq 0$, we have

$$
\begin{gathered}
G(s)=b s^{2}+s^{\beta}+(m+1) \log s, \\
G^{\prime}(s)=2 b s+\beta s^{\beta-1}+\frac{m+1}{s}, \\
G^{\prime \prime}(s)=2 b+\beta(\beta-1) s^{\beta-2}-\frac{m+1}{s^{2}}, \\
G(s)-\frac{1}{2} s G^{\prime}(s)=\left(1-\frac{\beta}{2}\right) s^{\beta}+(m+1) \log s-\frac{m+1}{2}, \\
\lim _{s \rightarrow \infty} \frac{s G^{\prime}(s)}{G(s)}=2, \quad \lim _{s \rightarrow \infty} \frac{s G^{\prime \prime}(s)}{G^{\prime}(s)}=1, \\
b s^{2} \leq G(s) \leq\left(b+1+\frac{m+1}{2 e}\right) s^{2} \quad \text { for } s \geq 1 .
\end{gathered}
$$

Since $\beta \leq 1$, from (2.29) we can choose an $s_{0}>0$ such that for all $s>s_{0}$, $G^{\prime \prime}(s) \geq 0$. Combining this with (2.27) to (2.32), $F$ satisfies all the assumptions 
from (2.18) to (2.22). Hence from Proposition 2, (2.28) and (2.30) we have as $\gamma \rightarrow \infty$,

$$
\begin{gathered}
Y^{\prime}\left(S_{0}(\gamma), \gamma\right)=O(1 / \gamma), \\
S_{0}(\gamma)=s_{0} b \gamma+O\left(\gamma^{\beta}\right), \\
T_{1}(\gamma)=S(\gamma) \geq(1-\beta / 2) \gamma^{\beta}+O(\log \gamma) .
\end{gathered}
$$

Hence $T_{1}(\gamma) \rightarrow \infty$ as $\gamma \rightarrow \infty$. This proves (2.8)' . Now from (2.34) and using $0 \leq \beta \leq 1$ we have for $\gamma$ large,

$$
\frac{T_{1}(\gamma)}{\gamma} \leq \frac{S_{0}(\gamma)}{\gamma} \leq s_{0} b+O\left(\gamma^{\beta-1}\right) \leq C_{3}
$$

for some constant $C_{3}>0$. This proves (2.8).

Let $C_{4}=\sup _{0 \leq s \leq s_{0}} F(s)$, then from (2.23) we have for $t \in\left[S(\gamma), S_{0}(\gamma)\right]$,

$$
-Y^{\prime \prime} \leq C_{4} e^{-t} \text {. }
$$

Integrating (2.37) from $T_{1}(\gamma) \quad(=S(\gamma))$ to $S_{0}(\gamma)$ we have

$$
Y^{\prime}\left(T_{1}(\gamma), \gamma\right) \leq Y^{\prime}\left(S_{0}(\gamma), \gamma\right)+C_{4}\left(e^{-T_{1}(\gamma)}-e^{-S_{0}(\gamma)}\right)
$$

Now from (2.33), (2.34) and (2.35), we can choose a constant $C_{5}>0$ such that for all $\gamma$ large, (2.38) implies $\gamma Y^{\prime}\left(T_{1}(\gamma), \gamma\right) \leq C_{5}$. This proves (2.7) and hence Claim 2.

Remark. The above proof shows that Theorem 1 can be stated in a more general form as follows.

Let $f: \overline{\mathbb{R}}_{+} \rightarrow \overline{\mathbb{R}}_{+}$be a $C^{1}$ function and let $s_{0} \geq 0$ be such that

$f$ is strictly increasing for $s \geq s_{0}$,

$$
\begin{gathered}
g(s)=\log f(s) \text { is } C^{2} \text { convex for } s \geq s_{0}, \\
g(s)=b s^{2}+g_{1}(s) \text { with } b>0 \text { such that } \\
\lim _{s \rightarrow \infty} \frac{g_{1}(s)}{s^{2}}=0, \quad \varlimsup_{s \rightarrow \infty}\left|g_{1}^{\prime}(s)\right|<\infty, \\
\lim _{s \rightarrow \infty} g_{1}^{\prime \prime}(s)=0, \quad \varlimsup_{s \rightarrow \infty} \frac{\left|g_{1}(s)-\frac{1}{2} s g_{1}^{\prime}(s)\right|}{s}<\infty .
\end{gathered}
$$

Then we have the following

Theorem 1'. Let $f$ satisfy (2.39), (2.40) and (2.41). Futher assume that $f(0)=$ 0 and extend $f$ as an odd function on $\mathbb{R}$. Then there exists an $R_{0}>0$ such that for $0<R<R_{0}$, (1.1) does not admit any radial solution which changes sign.

\section{APPENDIX}

Appendix 1. Let $F: \overline{\mathbb{R}}_{+} \rightarrow \overline{\mathbb{R}}_{+}$be a locally Lipschitz continuous function and $G(s)=\log F(s)$ satisfies (2.18) to (2.22). Following the same notations as in Proposition 2 and denoting $G(\gamma)=G, G^{\prime}(\gamma)=G^{\prime}, Y(t, \gamma)=Y(t)$, we have the following 
Lemma 3.1. For $S_{0} \leq t<\infty$ we have

$$
\begin{gathered}
Y(t) \leq \gamma-\frac{2}{G^{\prime}} \log \left(1+\frac{1}{2} G^{\prime} e^{G-t}\right), \\
G(Y(t)) \geq G-2 \log \left(1+\frac{1}{2} G^{\prime} e^{G-t}\right), \\
t>G-\frac{1}{2}(\gamma-Y(t)) G^{\prime}+\log \frac{G^{\prime}}{2}, \\
t \leq \frac{1}{2}\{G+G(Y(t))\}+\log \frac{G^{\prime}}{2}-\log \left[1-e^{\{G(Y(t))-G\} / 2}\right], \\
Y^{\prime}(t) \leq e^{\{(G+G(Y(t))) / 2-t\}}, \\
Y^{\prime}(t) \geq e^{\left\{G-(\gamma-Y(t)) G^{\prime} / 2-t\right\}}, \\
S(\gamma) \geq G-\frac{1}{2} \gamma G^{\prime}+\log \frac{G^{\prime}}{2}+O(1) \quad \text { as } \gamma \rightarrow \infty .
\end{gathered}
$$

For the proof of this lemma we refer to Atkinson-Peletier [2]. In fact (3.1), (3.2), (3.3), (3.4), (3.5), (3.6) and (3.7) of the above lemma correspond to (4.4), (4.5), (4.16), (4.18), (4.21), (4.22) and (3.5) of Atkinson-Peletier [2].

Let $k$ be a large positive (but fixed) number and define

$$
\begin{gathered}
\delta=k \log \gamma, \\
S_{1}=G+\log \frac{G^{\prime}}{2}-\delta .
\end{gathered}
$$

Then we have the following

Lemma 3.2. As $\gamma \rightarrow \infty$, we have

$$
\begin{gathered}
Y\left(S_{1}\right)=\gamma-\frac{2}{G^{\prime}} \delta+O\left(\frac{\delta^{2}}{G}\right), \\
G\left(Y\left(S_{1}\right)\right)=G-2 \delta+O\left(\frac{\delta^{2}}{G}\right), \\
Y^{\prime}\left(S_{1}\right)=\frac{2}{G^{\prime}}\left[1+O\left(\frac{\delta^{2}}{G}\right)\right] .
\end{gathered}
$$

Proof. Taking $t=S_{0}$ in (3.4) we have for large $\gamma$,

$$
\begin{aligned}
S_{0} & \leq G+\log \frac{G^{\prime}}{2}-\frac{1}{2} G+O(1) \\
& =S_{1}-\left(\frac{1}{2} G-\delta\right)+O(1)<S_{1} .
\end{aligned}
$$

Hence from (3.1) and (3.2), we have

$$
Y\left(S_{1}\right) \leq \gamma-\frac{2}{G^{\prime}} \log \left(1+e^{\delta}\right) \leq \gamma-\frac{2}{G^{\prime}} \delta+O\left(\frac{2}{G^{\prime}} e^{-\delta}\right)
$$


and

$$
G\left(Y\left(S_{1}\right)\right) \geq G-2 \delta+O\left(e^{-\delta}\right) .
$$

Since $G$ is an increasing function, we have from (3.14)

$$
\begin{aligned}
& G\left(Y\left(S_{1}\right)\right) \leq G\left(\gamma-\frac{2}{G^{\prime}} \delta+O\left(\frac{2}{G^{\prime}} e^{-\delta}\right)\right) \\
& =G-\left[\frac{2}{G^{\prime}} \delta+O\left(\frac{2}{G^{\prime}} e^{-\delta}\right)\right] G^{\prime}+\left[\frac{2 \delta}{G^{\prime}}+O\left(\frac{2}{G^{\prime}} e^{-\delta}\right)\right]^{2} \frac{G^{\prime \prime}(\xi)}{2}
\end{aligned}
$$

for some $\xi$ in the interval $\left[\gamma-2 \delta / G^{\prime}+O\left(2 e^{-\delta} / G^{\prime}\right), \gamma\right]$. Now from (2.21), we have $G^{\prime \prime}(\xi) /\left(G^{\prime}\right)^{2}=O(1 / G)$ and hence $(3.16)$ implies

$$
G\left(Y\left(S_{1}\right)\right) \leq G-2 \delta+O\left(\delta^{2} / G\right)
$$

Therefore from (3.15) we have

$$
G\left(Y\left(S_{1}\right)\right)=G-2 \delta+O\left(\delta^{2} / G\right)
$$

This proves (3.11).

From (3.15) we have

$$
\begin{aligned}
Y\left(S_{1}\right) & \geq G^{-1}\left(G-2 \delta+O\left(e^{-\delta}\right)\right) \\
& =\gamma-\frac{\left(2 \delta+O\left(e^{-\delta}\right)\right)}{G^{\prime}}-\frac{\left(2 \delta+O\left(e^{-\delta}\right)\right)^{2}}{2} \frac{G^{\prime \prime}(\eta)}{\left(G^{\prime}(\eta)\right)^{3}}
\end{aligned}
$$

for some $\eta$ such that

$$
G-2 \delta+O\left(e^{-\delta}\right) \leq G(\eta) \leq G .
$$

Now from (2.22) it follows that there exists a constant $C_{1}>0$ such that $C_{1} \gamma \leq$ $\eta \leq \gamma$. Therefore from (3.18) and (3.14) we have

$$
Y\left(S_{1}\right)=\gamma-\frac{2 \delta}{G^{\prime}}+O\left(\frac{\delta^{2}}{G}\right) .
$$

This proves (3.10).

Let $t=S_{1}$ in (3.5). Then using (3.11) we have

$$
Y^{\prime}\left(S_{1}\right) \leq e^{\left\{-\log G^{\prime} / 2+O\left(\delta^{2} / G\right)\right\}}=\frac{2}{G^{\prime}}\left[1+O\left(\frac{\delta^{2}}{G}\right)\right] .
$$

Similarly from (3.6) we obtain

$$
Y^{\prime}\left(S_{1}\right) \geq \frac{2}{G^{\prime}}\left[1+O\left(\frac{\delta^{2}}{G}\right)\right] .
$$

Combining (3.20) and (3.21) we get (3.12). This completes the proof of the lemma.

Lemma 3.3. For $S_{0} \leq t \leq S_{1}$, we have

$$
Y^{\prime}(t)=\frac{2}{G^{\prime}}\left[1+O\left(\frac{\delta^{2}}{G}\right)+O\left(\gamma G^{\prime} e^{-\left\{G-\frac{1}{2}\left(\gamma-s_{0}\right) G^{\prime}\right\}}\right)\right] .
$$

Proof. From (3.3) we have

$$
G(Y(t))-t \leq\left\{G(Y(t))-\frac{1}{2} Y(t) G^{\prime}\right\}-\left\{G-\frac{1}{2} \gamma G^{\prime}\right\}-\log \frac{G^{\prime}}{2} \equiv \psi(Y) .
$$


Since $\psi^{\prime \prime}(Y) \geq 0$, it follows that

$$
G(Y(t))-t \leq \max \left\{\psi\left(Y\left(S_{1}\right)\right), \psi\left(Y\left(S_{0}\right)\right)\right\} .
$$

Using (3.10) and (3.11), the above implies that

$$
G(Y(t))-t \leq-\log \frac{G^{\prime}}{2}+\max \left\{-\delta,-\left(G-\frac{1}{2}\left(\gamma-s_{0}\right) G^{\prime}\right)\right\}+O(1) .
$$

Hence from (3.12) and (3.24) we have for any $t \in\left[S_{0}, S_{1}\right]$

$$
\begin{aligned}
Y^{\prime}(t) & =Y^{\prime}\left(S_{1}\right)+\int_{t}^{S_{1}} e^{G(Y(s))-s} d s \\
& =\frac{2}{G^{\prime}}\left[1+O\left(\frac{\delta^{2}}{G}\right)\right]+O\left[\frac{S_{1}-S_{0}}{G^{\prime}} \max \left\{e^{-\delta}, e^{-\left(G-\frac{1}{2}\left(\gamma-s_{0}\right) G^{\prime}\right)}\right] .\right.
\end{aligned}
$$

From (2.20), $G-\frac{1}{2}\left(\gamma-s_{0}\right) G^{\prime}>0$ for $\gamma$ large, hence we have from (3.3), $S_{0} \geq \log G^{\prime} / 2$ which implies that $S_{1}-S_{0} \leq G$. Hence (3.25) implies

$$
\left.Y^{\prime}(t)=\frac{2}{G^{\prime}}\left[1+O\left(\frac{\delta^{2}}{G}\right)+O\left(\gamma G^{\prime} e^{-\left\{G-\frac{1}{2}\left(\gamma-s_{0}\right) G^{\prime}\right.}\right\}\right)\right] .
$$

This proves the lemma.

Proof of Proposition 2. (2.24) follows from Lemma 3.3. (2.26) follows from (3.7) of Lemma 3.1. Now from the mean value theorem, there exists a $t \in$ $\left[S_{0}, S_{1}\right]$ such that

$$
S_{0}=S_{1}-\frac{Y\left(S_{1}\right)-s_{0}}{Y^{\prime}(t)}
$$

From (3.10) and (3.22), (3.26) implies that

$$
\begin{aligned}
S_{0}= & S_{1}-\frac{\gamma-2 \delta / G^{\prime}+O\left(\delta^{2} / G\right)-s_{0}}{\left(2 / G^{\prime}\right)\left[1+O\left(\delta^{2} / G\right)+O\left(\gamma G^{\prime} e^{-\left\{G-\frac{1}{2}\left(\gamma-s_{0}\right) G^{\prime}\right\}}\right]\right.} \\
= & G-\frac{1}{2} \gamma G^{\prime}+\frac{s_{0} G^{\prime}}{2}+\log \frac{G^{\prime}}{2}+O\left(\frac{\gamma G^{\prime}}{G} \delta^{2}\right) \\
& +O\left(\left(\gamma G^{\prime}\right)^{2} e^{-\left\{G-\frac{1}{2}\left(\gamma-s_{0}\right) G^{\prime}\right\}}\right) .
\end{aligned}
$$

Since $O\left(\gamma G^{\prime} \delta^{2} / G\right)=O\left((\log \gamma)^{2}\right)$, (3.27) implies (2.25). This proves the proposition.

Appendix 2. Let $f: \overline{\mathbb{R}}_{+} \rightarrow \overline{\mathbb{R}}_{+}$be a $C^{1}$ function such that $f(0)=0$. Let $y(t, \gamma)$ be the solution of

$$
\left\{\begin{array}{l}
-y^{\prime \prime}=e^{-t} f(y), \\
y(\infty)=\gamma>0, \\
y^{\prime}(\infty)=0,
\end{array}\right.
$$

and $T_{1}(\gamma)$ the first zero of $y(t, \gamma)$. Then there exists a real number $C$ such that

$$
\lim _{\gamma \rightarrow 0} T_{1}(\gamma)= \begin{cases}-\infty & \text { if } f^{\prime}(0)=0 \\ C & \text { if } f^{\prime}(0) \neq 0\end{cases}
$$


Proof. (i) Let $f^{\prime}(0)=0$. Then integrating (3.28) from $T_{1}(\gamma)$ to $\infty$, we obtain

$$
\begin{aligned}
\gamma & =\int_{T_{1}(\gamma)}^{\infty}\left(s-T_{1}(\gamma)\right) e^{-s} f(y(s)) d s \\
& \leq \gamma e^{-T_{1}(\gamma)} \sup _{0 \leq y \leq \gamma} \frac{f(y)}{y} .
\end{aligned}
$$

This implies that

$$
e^{T_{1}(\gamma)} \leq \sup _{0 \leq y \leq \gamma} \frac{f(y)}{y} \rightarrow 0 \quad \text { as } \gamma \rightarrow 0 .
$$

Hence $T_{1}(\gamma) \rightarrow-\infty$ as $\gamma \rightarrow 0$.

(ii) Let $f^{\prime}(0)>0$. Let $\varphi$ be the solution of

$$
\left\{\begin{array}{l}
-\varphi^{\prime \prime}=f^{\prime}(0) e^{-t} \varphi \\
\varphi(\infty)=1 \\
\varphi^{\prime}(\infty)=0
\end{array}\right.
$$

and $T$ the first zero of $\varphi$.

For any two nonnegative continuous functions $\rho_{1}$ and $\rho_{2}$ defined on $\mathbb{R}$ with $\rho_{1} \geq \rho_{2}$, consider the following problem $\pi_{i}(i=1,2)$.

$$
\left(\pi_{i}\right):\left\{\begin{array}{l}
-\psi^{\prime \prime}=\rho_{i} e^{-t} \psi \quad \text { in } \mathbb{R} \\
\psi(\infty)>0 \\
\psi^{\prime}(\infty)=0
\end{array}\right.
$$

Let $\psi_{i}$ be a solution of $\left(\pi_{i}\right)$ and $T_{i}$ the first zero of $\psi_{i}$. Then we claim that

$$
T_{2} \leq T_{1} .
$$

Suppose not, then $T_{1}<T_{2}$ and let $W(t)=\psi_{1} \psi_{2}^{\prime}-\psi_{2} \psi_{1}^{\prime}$. Then $W^{\prime}(t)=$ $\psi_{1} \psi_{2} e^{-t}\left(\rho_{1}-\rho_{2}\right)$ and hence integrating $W^{\prime}(t)$ from $T_{2}$ to $\infty$, we have

$$
-\psi_{1}\left(T_{2}\right) \psi_{2}^{\prime}\left(T_{2}\right)=\int_{T_{2}}^{\infty} \psi_{1} \psi_{2} e^{-t}\left(\rho_{1}-\rho_{2}\right) d t \geq 0
$$

which is a contradiction. This proves (3.32).

Now for every $\varepsilon>0$, there exists a $\delta>0$ such that for $0<y<\delta$,

$$
(1-\varepsilon) f^{\prime}(0) \leq f(y) / y \leq(1+\varepsilon) f^{\prime}(0),
$$

Let $0<\gamma<\delta$ and by taking

$$
\rho_{2}=(1-\varepsilon) f^{\prime}(0), \quad \rho_{1}=\frac{f(y)}{y}, \quad \psi_{2}(t)=\varphi\left(t+\log \frac{1}{1-\varepsilon}\right),
$$

we obtain from (3.32) and (3.33),

$$
T+\log (1-\varepsilon) \leq T(\gamma) .
$$

Similarly by taking

$$
\rho_{1}=(1+\varepsilon) f^{\prime}(0), \quad \psi_{1}=\varphi\left(t+\log \frac{1}{1+\varepsilon}\right), \quad \rho_{2}=\frac{f(y)}{y},
$$

we obtain

$$
T(\gamma) \leq T+\log (1+\varepsilon) .
$$

Since $\varepsilon$ is arbitrary, from (3.34) and (3.35) we obtain $\lim _{\gamma \rightarrow 0} T(\gamma)=T$. This proves (3.29). 


\section{REFERENCES}

1. Adimurthi and S. L. Yadava, Multiplicity results for semilinear elliptic equations in a bounded domain of $\mathbb{R}^{2}$ involving critical exponents, Ann. Scuola Norm. Sup. Pisa Cl. Sci. 27 (1990), 481-504.

2. F. V. Atkinson and L. A. Peletier, Ground states and Dirichlet problem for $-\Delta u=f(u)$ in $\mathbb{R}^{2}$, Arch. Rational Mech. Anal. 96 (1986), 147-165.

3. __ Emden-Fowler equations involving critical exponents, Nonlinear Anal. TMA 10 (1986), 755-776.

4. F. V. Atkinson, H. Brezis and L. A. Peletier, Nodal solutions of elliptic equations with critical Sobolev exponents, C. R. Acad. Sci. Paris 306 (1988), 711-714.

5. G. Cerami, S. Solomini and M. Struwe, Some existence results for super linear elliptic boundary value problems involving critical exponents, J. Functional Anal. 69 (1986), 289306.

T. I. F. R. Centre, Post BoX No. 1234, Bangalore-560 012, India 